\title{
Inhaled chemotherapy in lung cancer: safety concerns of nanocomplexes delivered
}

\author{
"No definite conclusions can be drawn regarding clearance of inhaled nanoparticles in the lungs, as this \\ is highly dependent on material characteristics, as well as the model examined."
}

Keywords: health safety $\approx$ inhaled chemotherapy $₫$ nanoparticles $\approx$ toxicity

The implementation of nanotechnology in targeted drug delivery is a promising and fast growing area. By definition the term 'nanomaterial' characterizes materials that have at least one dimension measuring $100 \mathrm{~nm}$ or less [1,2]. Critical parameters for their characterization, other than size, may include shape, ratio of surface area to mass, material composition and functional phenomena. However, the size of therapeutic nanomaterial ranges from 2 to $1000 \mathrm{~nm}[3,4]$. The major substances used in drug 'nanodelivery' are liposomes, polymers, micelles and dendrimers [5].

The inhaled route for drug administration has been used for several drug formulations, not only in respiratory diseases, but also in systemic diseases (diabetes mellitus) [6]. Since previous inhaled agents, therapies for systemic diseases have been approved and are already on the market; other systemic therapies, such as inhaled chemotherapy in lung cancer, are under in vitro and in vivo evaluation [7]. A major concern regarding this route of administration is the safety of chemotherapeutic agents for lung parenchyma.

Thanks to the progress in nanotechnology, 'nanocomplexes' and 'nanocarriers' are being created to exploit the characteristics of nanoparticles and deliver high concentrations of chemotherapeutic agents to the preferred site. These predominantly use liposomes and silica for the transport, and receptor-ligand technology for selective delivery (EGF, $\alpha$-tocopherol etherlinked acetic acid and luteinizing hormonereleasing hormone peptide) [8,9]. Particle size is a key factor for the deposition of aerosol particles in the human respiratory tract [10]. The formulation of aerosolized nanocomplexes, apart from enhancing the deposition in the alveolar region, provide properties such as local controlled sustain release and augment the drug formulation efficiency by targeting specific receptor-ligand groups using peptides on the nanocarrier surface
(EGF, $\alpha$-tocopherol ether-linked acetic acid and luteinizing hormone-releasing hormone peptide) [6,11].

The efficacy and safety of such approaches have been evaluated in vitro and in vivo for several chemotherapeutic drugs, either in their commercial or nanocomplexed forms [7,11-18]. The major advantages of inhaled chemotherapeutic agents include reduced systematic side effects and efficient control of micrometastasis. These are attributable to the circulation of chemotherapeutic agents through the blood or lymphatic route, after being absorbed from the alveoli and alveolar macrophages $[19,20]$.

However, a major concern has arisen during the in vitro and in vivo experiments. The adverse respiratory effect observed was an acute form of dose-dependent pneumonitis, which, in some cases, induced pulmonary edema, alveolar hemorrhage and death in animals [13,21]. These adverse effects were recorded in very low numbers and were not observed in human patients, possibly as a result of the different structure and defense mechanisms of the human respiratory system [7,11,12]. Respiratory evaluation in patients has predominantly included respiratory function tests (e.g., forced expiratory volume in $1 \mathrm{~s}$, forced vital capacity, diffusing capacity of the lung for $\mathrm{CO}$ and peak expiratory flow) and bronchoalveolar lavage. It was observed that inhaled corticosteroid administration and bronchodilators protected patients from symptoms, notably bronchospasm and cough. Nevertheless, in some cases, fever remained for a maximum of 3 days and was dose dependent $[7,11]$. Safety concerns regarding the medical staff urged the investigators to perform the inhalation administration inside a high-efficiency particulate air system and evaluate whether any inhaled aerosol was not properly absorbed or administered [12]. In this way, the minimal concentrations of escaped aerosol were

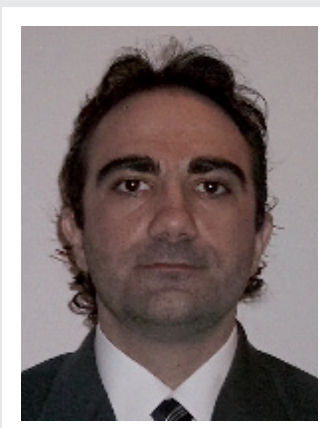

Paul Zarogoulidis

Author for correspondence: Pulmonary Department, G Papanikolaou General Hospital, Aristotle University of Thessaloniki, Greece

Tel.: +306977271974

Fax: +30231099 2433

E-mail: pzarog@hotmail.com

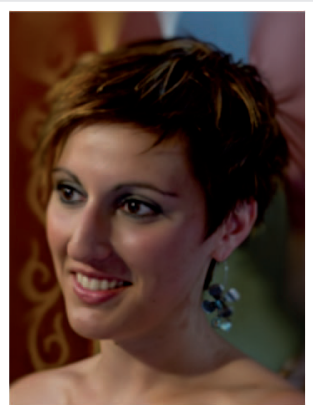

Chrisostomi Gialeli

Laboratory of Biochemistry, Department of Chemistry, University of Patras, Greece and

Foundation for Research \& Technology - Hellas/Institute of Chemical Engineering Sciences, Greece

Authors continued overleaf..

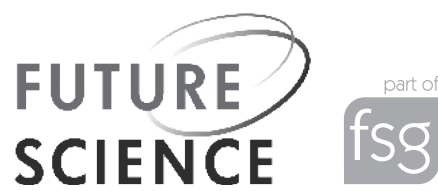




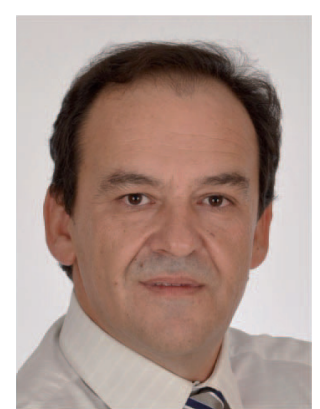

Nikos K Karamanos

Laboratory of Biochemistry, Department of Chemistry, University of Patras, Greece and

Foundation for Research and Technology - Hellas/Institute of Chemical Engineering Sciences, Greece measured. It is important to remember that the latter also depends on drug system administration (nebulizer mouthpiece), so conclusions cannot be drawn until several systems are evaluated. Furthermore, the microenvironment interactions with the inhaled regimen should be extensively evaluated [22,23].

No definite conclusions can be drawn regarding clearance of inhaled nanoparticles in the lungs, as this is highly dependent on material characteristics, as well as the model examined. So far, studies have shown long-term retention of nanoparticles in human airways and no more than $1 \%$ translocation of the nanoparticle dose delivered, in contrast with rodents, in which $20-30 \%$ of nanoparticles rapidly cleared from the lungs [23].

Moreover, before going from the 'lab bench' to 'actual' human inhalation exposure, nanomaterials must undergo extensive physicochemical characterization (particle shape, surface area/energy/chemistry/charge and state of dispersion/agglomeration), as well as assessment of several toxicological and ecotoxicological endpoints. A variety of in vitro, in vivo and ex vivo techniques have been established for the evaluation of inhaled nanoparticles at the level of particle size distribution, pharmacokinetics, as well as mechanisms of drug transport and disposition. Such techniques involve laser and imaging experimental procedures; classical bioassays, to establish pharmacokinetics and dynamics in animal models; clinical trials; and ex vivo isolated, perfused and ventilated lung models [24]. In vitro toxicological assessment should pay attention to the delivery systems affecting the administration routes - in this case, mainly lung tissue (human lung epithelial/fibroblasts cells) and, secondarily, blood and the gastrointestinal system. Nanomaterials are so small that they are even able to reach the nuclei of cells. Importantly, they may also interact with molecules on the cell surface and within the cell. Toxicological endpoints involve cytotoxicity studies; first at the level of cell viability assays, evaluating dose- and time-dependent (short- and long-term evaluation) effects via mitochondrial activity and DNA stain, as well as cellular uptake reflecting activity, phenotype and health, using fluorescent tags. Toxicity related with oxidative stress and inflammatory defense response induced by nanomaterials should be examined via production of inflammatory cytokines (IL-1b, IL-6, IL-8 and TNF- $\alpha$ ), as well as cell via alterations in the cell cycle. The latter should include cell characterization (i.e., healthy, dead, apoptotic and necrotic). At a deeper level, in vitro genotoxicity evaluation is the next step in assaying cell cytoskeleton and microtubule network along with DNA damage. Detection of DNA damage is conducted via comet assay, following treatment of blood cells with nanoparticles, encapsulation of cells in an agarose suspension, electrophoresis of lysed cells and DNA staining. The hemolytic potential of red blood cells upon exposure with nanoparticles may be also assessed, measuring the amount of hemoglobin released [25]. Upon human exposure, administration of inhaled nanoparticles must not only be evaluated in lung parenchyma, but also at the level of its potential translocation to the adjacent microenvironment. Toxicity should be assessed both in patients and medical personnel. Blood and urine samples may be analyzed for inflammatory cytokines and other biologic markers.

\section{"The major factor affecting aerosol or dry powder efficacy is the drug-delivery system. Once this is found, the next step should be to perform studies whereby patients receive their treatment at home."}

The major advances required for this treatment modality can be summarized as follows: the creation of a universal model for aerosolized treatment trials evaluation; trials with inhaled agents only; evaluation of safety via inflammatory markers other than histological, but also with high-resolution computed scans; and education of patients to receive treatment at home. Inhaled chemotherapy offers the additional advantage of easy use for patients educated in how to administer the aerosol. The major factor affecting aerosol or dry powder efficacy is the drug-delivery system. Once this is found, the next step should be to perform studies whereby patients receive their treatment at home. In these patients, respiratory capacity should be measured with peak expiratory flow measurements before and after treatment. In cases of unexpected deterioration, their physician should be alerted, made possible by an online system. Cost-effectiveness of this approach in comparison with standard treatment can be increased based on reduced hospitalization. In addition, reduced systemic toxicity is expected, leading to improved health and, again, reduced hospitalization rates. We now need further trials to progress towards providing an alternative efficient, safe, cost-effective and easy-to-use treatment modality for lung cancer patients. 


\section{Financial \& competing interests disclosure}

The authors have no relevant affliations or financial involvement with any organization or entity with a financial interest in or financial conflict with the subject matter or materials discussed in the manuscript. This includes employment, consultancies, honoraria, stock ownership or options, expert testimony, grants or patents received or pending, or royalties.

No writing assistance was utilized in the production of this manuscript.

\section{References}

1 Maynard AD. Don't define nanomaterials. Nature 475 (7354), 31 (2011).

2 US FDA. Reporting Format for Nanotechnology-Related Information in CMC Review. Office of Pharmaceutical Science, Silver Spring, MD, USA (2010).

3 Bawarski WE, Chidlowsky E, Bharali DJ, Mousa SA. Emerging nanopharmaceuticals. Nanomedicine 4(4), 273-282 (2008).

4 Brouwer N, Weda M, van Riet-Nales D, de Kaste D. Nanopharmaceuticals: implications for the European pharmacopoeia. Pharmeuropa 22, 5-7 (2010).

5 Zhang L, Gu FX, Chan JM, Wang AZ, Langer RS, Farokhzad OC. Nanoparticles in medicine: therapeutic applications and developments. Clin. Pharmacol. Ther. 83(5), 761-769 (2008).

6 Zarogoulidis P, Papanas N, Kouliatsis G, Spyratos D, Zarogoulidis K, Maltezos E. Inhaled insulin: too soon to be forgotten? J. Aerosol Med. Pulm. Drug Deliv. 24(5), 213-223 (2011).

7 Zarogoulidis P, Eleftheriadou E, Sapardanis I et al. Feasibility and effectiveness of inhaled carboplatin in NSCLC patients. Invest. New Drugs 30(4), 1628-1640 (2011).
8 Lebhardt T, Roesler S, Uusitalo HP, Kissel T. Surfactant-free redispersible nanoparticles in fast-dissolving composite microcarriers for dry-powder inhalation. Eur. J. Pharm. Biopharm. 78(1), 90-96 (2011).

9 Taratula O, Garbuzenko OB, Chen AM, Minko T. Innovative strategy for treatment of lung cancer: targeted nanotechnology-based inhalation co-delivery of anticancer drugs and siRNA. J. Drug Target. 19(10), 900-914 (2011).

10 Yang W, Peters JI, Williams RO III. Inhaled nanoparticles - a current review. Int. J. Pharm. 356(1-2), 239-247 (2008).

11 Tseng CL, Su WY, Yen KC, Yang KC, Lin FH. The use of biotinylated EGF-modified gelatin nanoparticle carrier to enhance cisplatin accumulation in cancerous lungs via inhalation. Biomaterials 30(20), 3476-3485 (2009).

12 Tatsumura T, Koyama S, Tsujimoto M, Kitagawa M, Kagamimori S. Further study of nebulisation chemotherapy, a new chemotherapeutic method in the treatment of lung carcinomas: fundamental and clinical. Br. J. Cancer 68 (6), 1146-1149 (1993).

13 Gautam A, Koshkina N. Paclitaxel (taxol) and taxoid derivates for lung cancer treatment: potential for aerosol delivery. Curr. Cancer Drug Targets 3(4), 287-296 (2003).

14 Otterson GA, Villalona-Calero MA, Hicks W et al. Phase I/II study of inhaled doxorubicin combined with platinum-based therapy for advanced non-small cell lung cancer. Clin. Cancer Res. 16(8), 2466-2473 (2010).

15 Wittgen BP, Kunst PW, Perkins WR, Lee JK, Postmus PE. Assessing a system to capture stray aerosol during inhalation of nebulized liposomal cisplatin. J. Aerosol Med. 19(3), 385-391 (2006).

16 Anderson K, Lawson KA, SimmonsMenchaca M, Sun L, Sanders BG, Kline K. Alpha-TEA plus cisplatin reduces human cisplatin-resistant ovarian cancer cell tumor burden and metastasis. Exp. Biol. Med. (Maywood) 229(11), 1169-1176 (2004).

17 El-Gendy N, Berkland C. Combination chemotherapeutic dry powder aerosols via controlled nanoparticle agglomeration. Pharm. Res. 26(7), 1752-1763 (2009).

18 Zarogoulidis P, Chatzaki E, Porpodis K et al. Inhaled chemotherapy in lung cancer: future concept of nanomedicine. Int. J. Nanomed. 7 , 1551-1572 (2012).

19 Labiris NR, Dolovich MB. Pulmonary drug delivery. Part I: physiological factors affecting therapeutic effectiveness of aerosolized medications. Br. J. Clin. Pharmacol. 56(6), 588-599 (2003).

20 Margaris KN, Black RA. Modelling the lymphatic system: challenges and opportunities. J. R. Soc. Interface 9(69), 601-612 (2012).

21 Selting K, Waldrep JC, Reinero C et al. Feasibility and safety of targeted cisplatin delivery to a select lung lobe in dogs via the AeroProbe intracorporeal nebulization catheter. J. Aerosol Med. Pulm. Drug Deliv. 21(3), 255-268 (2008).

22 Bosquillon C. Drug transporters in the lung - do they play a role in the biopharmaceutics of inhaled drugs? J. Pharm. Sci. 99(5), 2240-2255 (2010).

23 Minchinton AI, Tannock IF. Drug penetration in solid tumours. Nat. Rev. Cancer 6(8), 583-592 (2006).

24 Zhang J, Wu L, Chan HK, Watanabe W. Formation, characterization, and fate of inhaled drug nanoparticles. Adv. Drug Deliv. Rev. 63(6), 441-455 (2011).

25 Arora S, Rajwade JM, Paknikar KM. Nanotoxicology and in vitro studies: the need of the hour. Toxicol. Appl. Pharmacol. 258(2), 151-165 (2012). 